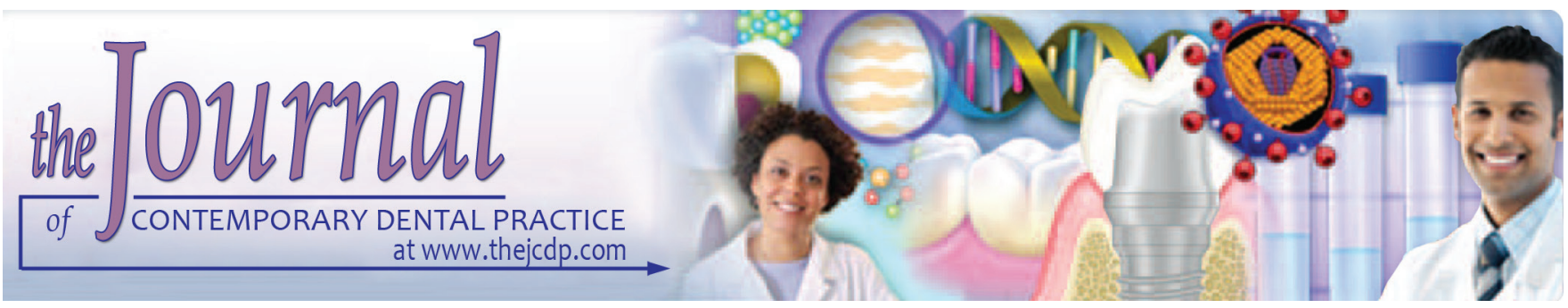

\title{
Effect of Three Different Injection Sites on the Success of Anterior Middle Superior Alveolar Nerve Block with 3\% Mepivacaine: A Randomized Controlled Trial
}

${ }^{1}$ Ali Shokraneh, ${ }^{2}$ Nastaran Farhadi, ${ }^{3}$ Masoud Saatchi, ${ }^{4}$ Hooman Navaei, ${ }^{5}$ Masoud Yaghmaei

\begin{abstract}
Aim: Anterior middle superior alveolar (AMSA) nerve block injection targets the anterior superior alveolar nerve and the middle superior alveolar nerve branches of the infraorbital nerve through nutrient canals. Therefore, the central incisor to the second premolar teeth of one quadrant can be anesthetized. The aim of the present study was to evaluate the efficacy of AMSA nerve block injection with $3 \%$ mepivacaine solution at three different injection sites.
\end{abstract}

Materials and methods: In a double-blind crossover study, 47 volunteers participated and three AMSA nerve block injections of $3 \%$ mepivacaine solution without epinephrine were administered at the anterior, posterior, and the most common injection sites with a 1-week interval between injections. Anesthesia of the central incisor to the second premolar of the injected side was evaluated by using an electric pulp tester. The success of the injection was considered as lack of response to two consecutive 80 readings. The generalized estimating equation analytic tests were administered $(\alpha=0.05)$.

Results: The success rate of the AMSA nerve block injection ranged from $27.5-47.5 \%$ for the most common injection site

\footnotetext{
${ }^{1}$ Department of Endodontics, School of Dentistry, Kashan University of Medical Sciences, Kashan, Islamic Republic of Iran

${ }^{2}$ Department of Oral and Maxillofacial Radiology, School of Dentistry, Physiology Research Center, Ahvaz Jundishapur University of Medical Sciences, Ahvaz, Islamic Republic of Iran

${ }^{3}$ Department of Endodontics, Torabinejad Dental Research Center, Isfahan University of Medical Sciences, Isfahan, Islamic Republic of Iran

${ }^{4}$ Department of Oral and Maxillofacial Surgery, School of Dentistry, Shiraz University of Medical Sciences, Shiraz Islamic Republic of Iran

${ }^{5}$ Dental Research Center, Research Institute of Dental Sciences, School of Dentistry, Shahid Beheshti University of Medical Sciences, Tehran, Islamic Republic of Iran

Corresponding Author: Nastaran Farhadi, Department of Oral and Maxillofacial Radiology, School of Dentistry, Ahvaz Jundishapur University of Medical Sciences, Ahvaz, Islamic Republic of Iran, e-mail:nastaranfarhadi@yahoo.com
}

and $22.5-42.5 \%$ for both the anterior and posterior injection sites.

Conclusion: Changing the injection site did not result in statistically significant improvements $(p>0.05)$.

Clinical significance: Changing the injection site anteroposteriorly did not influence the success rate of the AMSA nerve block injection.

Keywords: Dental pulp, Local anesthesia, Mepivacaine, Nerve block, Randomized controlled trial.

How to cite this article: Shokraneh A, Farhadi N, Saatchi M, Navaei H, Yaghmaei M. Effect of Three Different Injection Sites on the Success of Anterior Middle Superior Alveolar Nerve Block with 3\% Mepivacaine: A Randomized Controlled Trial. J Contemp Dent Pract 2016;17(2):130-135.

Source of support: Nil

Conflict of interest: None

\section{INTRODUCTION}

Local anesthesia of maxillary teeth is commonly achieved by the local infiltrative supraperiosteal injections next to the apex of the tooth under question in the buccal vestibule. $^{1-3}$ In the anterior and posterior teeth, infiltration anesthesia results in a high incidence (90-95\%) of successful pulpal anesthesia. ${ }^{4,5}$ In addition, supplementary injections, such as the nerve block, periodontal ligament, intraosseous, intrapulpal, and palatal injections can be administered. ${ }^{3,6}$ A negative consequence of traditional maxillary injections is their effect on muscles of facial expression and labial muscles. ${ }^{7}$

Friedman and Hochman ${ }^{8}$ introduced a new injection technique, called the "anterior middle superior alveolar (AMSA) nerve block" injection, which anesthetizes the anterior and middle superior alveolar branches of the infraorbital nerve. This injection is administrated palatally at a point that bisects the premolars and is approximately 
halfway between the midpalatine raphe and the crest of the free gingival margin. In this site, the anterior superior alveolar and middle superior alveolar nerve branches join together as a plexus. The anterior superior alveolar nerve innervates the central and lateral incisors and the canine tooth and the middle superior alveolar nerve innervates the premolars and the mesiobuccal root of the first molar teeth. ${ }^{9-13}$ In this technique, $0.6-0.9 \mathrm{ml}$ of the anesthetic solution is injected supraperiosteally in 60-90 seconds in the palate as a single injection. ${ }^{8,14}$ This injection can anesthetize the central incisor to the second premolar teeth of one side of the maxilla for 45-60 minutes. ${ }^{8,14}$ With a bilateral AMSA nerve block injection, ten maxillary teeth from the second premolar of a quadrant to the second premolar of the adjacent quadrant can be anesthetized without any interference with the patient's expression muscles and smile line. ${ }^{8}$

In the infiltration technique, the anesthetic solution should pass through the periosteum and porous maxillary bone to reach the neurovascular bundles associated with the teeth. ${ }^{15}$ However, in the AMSA nerve block injection, the anesthetic solution reaches the bundles from the injection site through the nutrient canals, which are accessible in the hard palate. ${ }^{14}$ The AMSA nerve block injection may be more effective in areas where the nutrient canals are more common; however, there is a lack of data about areas where the nutrient canals are common. The aim of the present study was to evaluate the efficacy of the AMSA nerve block injection at anterior and posterior areas compared with the most common injection site.

\section{MATERIALS AND METHODS}

This study was approved by the Ethics Committee of the Dental Research Center of Shahid Beheshti University of Medical Sciences (9117/17) and registered at www.clinical trial.gov (ClinicalTrials.gov identifier: NCT02012257). A sample size of 31 was calculated to provide $90 \%$ power $(\alpha=0.05)$ by using power analysis and sample size software for Windows (NCSS Statistical Software, Kaysville, USA).

This double-blind crossover study was performed on 47 volunteers, referring to the Dental School of Shahid Beheshti University of Medical Sciences. All the subjects were healthy and did not take any medications alleviating or altering the pain sensation, like narcotics, sedatives, antianxiety or antidepressant agents. Allergy to anesthetics and pregnancy were other exclusion criteria. All the subjects had all their maxillary teeth from the second premolar of one side to the second premolar of another. Neither of these teeth was nonvital nor had large restorations, prosthetic crowns, caries, periodontal diseases, history of trauma, and hypersensitivity. The side effects of the injection, expectancy of pain, and consequences of the injection were explained to each participant. Informed consent was obtained from all the subjects.

Before injection, the table of random numbers was used to select the side of the maxilla for injection. The central incisor to premolar teeth of the selected side of the maxilla, in addition to the lower canine of the same side, were tested by an electric pulp tester (EPT; Analytic Technology, Redmond, USA) to assess the vitality and response at baseline. Each tooth was dried and isolated by a cotton roll and toothpaste was placed on the EPT tip, which was placed right on the incisal or occlusal third of the buccal surface of the tooth. Then, the output of EPT increased in 30 seconds from 0 to 80 , and the result was recorded.

Before this study, a pilot study was carried out on some patients to identify potential problems during the main study and make preparations for the accurate process. The injections for each subject were administered on three different areas of the hard palate. Injection site was chosen from a table of random numbers, while the subjects were unaware of the injection site. A week after each injection, the participants were summoned for the next injection (Flow Chart 1). Injection " $A$ " was administered at an anterior site which was palatally at a point bisecting the canine and the first premolar, approximately halfway between the midpalatine raphe and the crest of the free gingival margin. Injection " $\mathrm{B}$ " was administered at the most commonly used AMSA injection

Flow Chart 1: A flow diagram of the study design according to the CONSORT statement

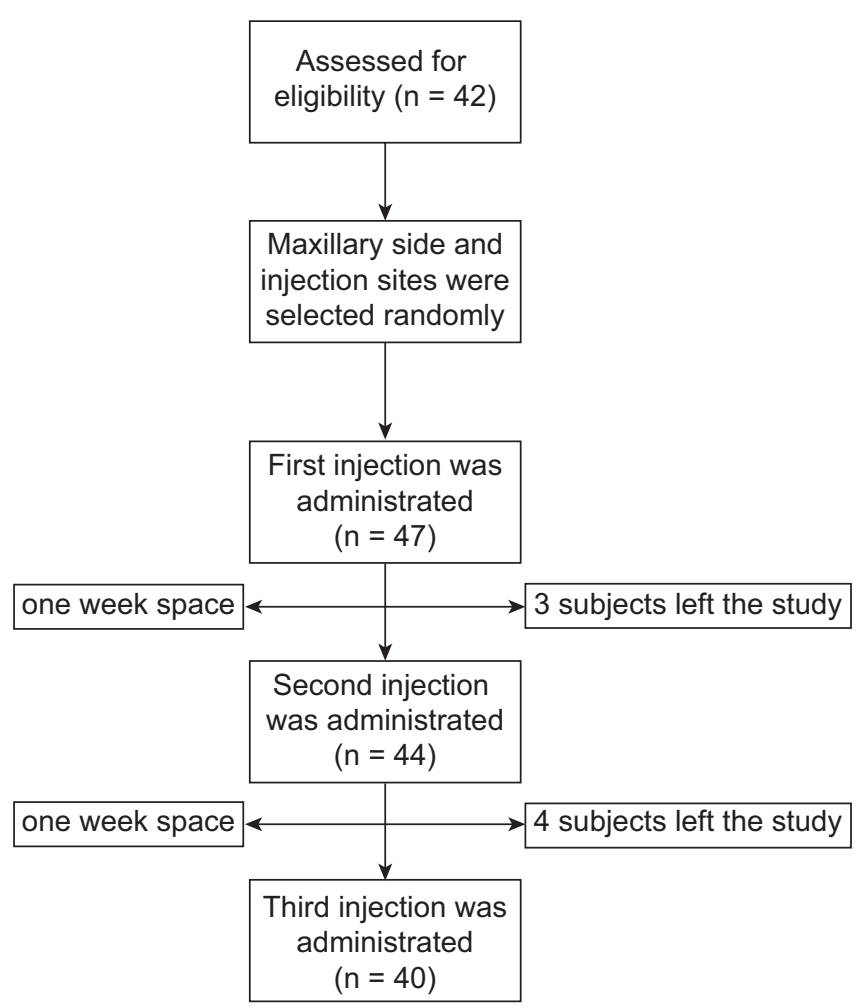


site which was palatally at a point bisecting the premolars, approximately halfway between the midpalatine raphe and the crest of the free gingival margin. Injection " $C$ " was administered at a posterior site which was palatally at a point bisecting the second premolar and the first molar, approximately halfway between the midpalatine raphe and the crest of the free gingival margin. All the injections were administered by an experienced endodontist (AS). Thus, with a crossover design, a total of 120 AMSA nerve block injections were administered in 40 subjects.

The palatal gingiva was anesthetized topically for about 1 minute by placing a cotton-tip applicator soaked in $0.2 \mathrm{ml}$ of benzocaine. A 27-gauge 1-inch needle (Monoject, Sherwood Medical, St Louis, USA) and $1.8 \mathrm{ml}$ 3\% mepivacaine solution without epinephrine (ESPE, Dental AG, Seefeld, Germany) were used. Each subject was told that the injection would take about $4-5$ minutes. In the insertion phase, the bevel of the needle was turned toward the soft tissue in order to enter the needle without soft tissue laceration and a cotton-tip applicator was pushed against the insertion site before and during this phase. The plunger of the syringe was pushed slowly to deposit the local anesthetic solution into the tissue, and the needle was inserted $1-2 \mathrm{~mm}$ forward. While continuing the deposition of the solution, the needle was inserted another 2-4 mm until it touched the bone. Then, the injection continued for 3-4 minutes up to complete deposition of the solution. Moreover, in order to decrease the side effects of this injection, whitening of the palatal mucosa was observed to prevent ischemia of the region. The depth of anesthesia was assessed using EPT. During the 1 st minute the second premolar, during the 2nd minute the first premolar, during the 3rd minute the canine, during the 4th minute the lateral incisor, during the 5th minute the central incisor, and during the 6th minute the lower canine were tested. This cycle was repeated ten times (a total of 60 minutes). The absence of reaction to the EPT was interpreted as complete anesthesia of the tooth. Anesthesia was considered successful if no reaction was observed to the maximum output in two consecutive tests. All the pulp tests were performed by a general practitioner $(\mathrm{HN})$.

Data were analyzed by Statistical Package for the Social Sciences (SPSS) 16.0 (SPSS Inc., Chicago, USA). The Cochran $\mathrm{Q}$ and the generalized estimating equation statistical tests were applied. The level of significance was set at 0.05 .

\section{RESULTS}

Of 47 subjects participating in the present study, three and four subjects did not continue cooperation after the first and second trials, respectively, and left the study due to intolerable pain during injection. Therefore, 40 subjects with 120 AMSA nerve block injections were included for statistical analysis. A total of 22 subjects were male and 18 were female, with a mean age of $26.7 \pm 3.2$ years. The injections were administered on the right side of the maxilla in 23 subjects and on the left side in 17 .

The success rates of the AMSA nerve block injections in the sites A, B, and C are presented in Table 1 and the pulpal anesthesia for each tooth is separately shown in graph 1. No significant differences were observed in success rates between the three sites of the AMSA nerve block injection for each tooth $(\mathrm{p}>0.05)$ (Table 2$)$.

Table 1: The percentages of the success rates of the AMSA nerve block injections at sites $A, B$, and $C$

\begin{tabular}{llll}
\hline & \multicolumn{3}{c}{ Injection site } \\
\cline { 2 - 4 } Teeth & $A$ & $B$ & $C$ \\
\hline Central incisor & $27.5 \%(11 / 40)$ & $27.5 \%(11 / 40)$ & $22.5 \%(9 / 40)$ \\
Lateral incisor & $42.5 \%(17 / 40)$ & $47.5 \%(19 / 40)$ & $27.5 \%(11 / 40)$ \\
Canine & $40 \%(16 / 40)$ & $37.5 \%(15 / 40)$ & $30 \%(12 / 40)$ \\
First premolar & $30 \%(12 / 40)$ & $27.5 \%(11 / 40)$ & $32.5 \%(13 / 40)$ \\
Second & $22.5 \%(9 / 40)$ & $37.5 \%(15 / 40)$ & $22.5 \%(9 / 40)$ \\
premolar & & & \\
\hline
\end{tabular}

A: Injection at a point bisecting the canine and the first premolar halfway between the midpalatine raphe and the crest of the free gingival margin; B: Injection at a point bisecting the premolars, halfway between the midpalatine raphe and the crest of the free gingival margin; C: Injection at a point bisecting the second premolar and the first molar, halfway between the midpalatine raphe and the crest of the free gingival margin; AMSA: anterior middle superior alveolar

Table 2: Generalized estimation equation statistical test for the efficacy of all the three injection sites on pulpal anesthesia (odds ratio is adjusted by age and gender)

\begin{tabular}{lllllllll}
\hline Teeth & Odds ratio A/B & LCB (95\%) & UCB (95\%) & $p$-value & Odds ratio C/B & LCB (95\%) & UCB (95\%) & $p$-value \\
\hline Central & 1.00 & 0.42 & 2.33 & 1.00 & 0.76 & 0.27 & 2.18 & 0.61 \\
Lateral & 0.81 & 0.35 & 1.89 & 0.63 & 0.41 & 0.15 & 1.12 & 0.08 \\
Canine & 1.10 & 0.45 & 2.71 & 0.82 & 0.71 & 0.30 & 1.66 & 0.44 \\
First premolar & 1.41 & 0.58 & 3.42 & 0.44 & 1.27 & 0.47 & 3.32 & 0.64 \\
Second premolar & 0.48 & 0.2 & 1.16 & 0.10 & 1.23 & 0.47 & 3.22 & 0.67 \\
\hline
\end{tabular}

A: Injection at a point bisecting the canine and the first premolar, halfway between the midpalatine raphe and the crest of the free gingival margin; B: Injection at a point bisecting the premolars, halfway between the midpalatine raphe and the crest of the free gingival margin; C: Injection at a point bisecting the second premolar and the first molar, halfway between the midpalatine raphe and the crest of the free gingival margin; LCB: lower confidence bound; UCB: upper confidence bound 


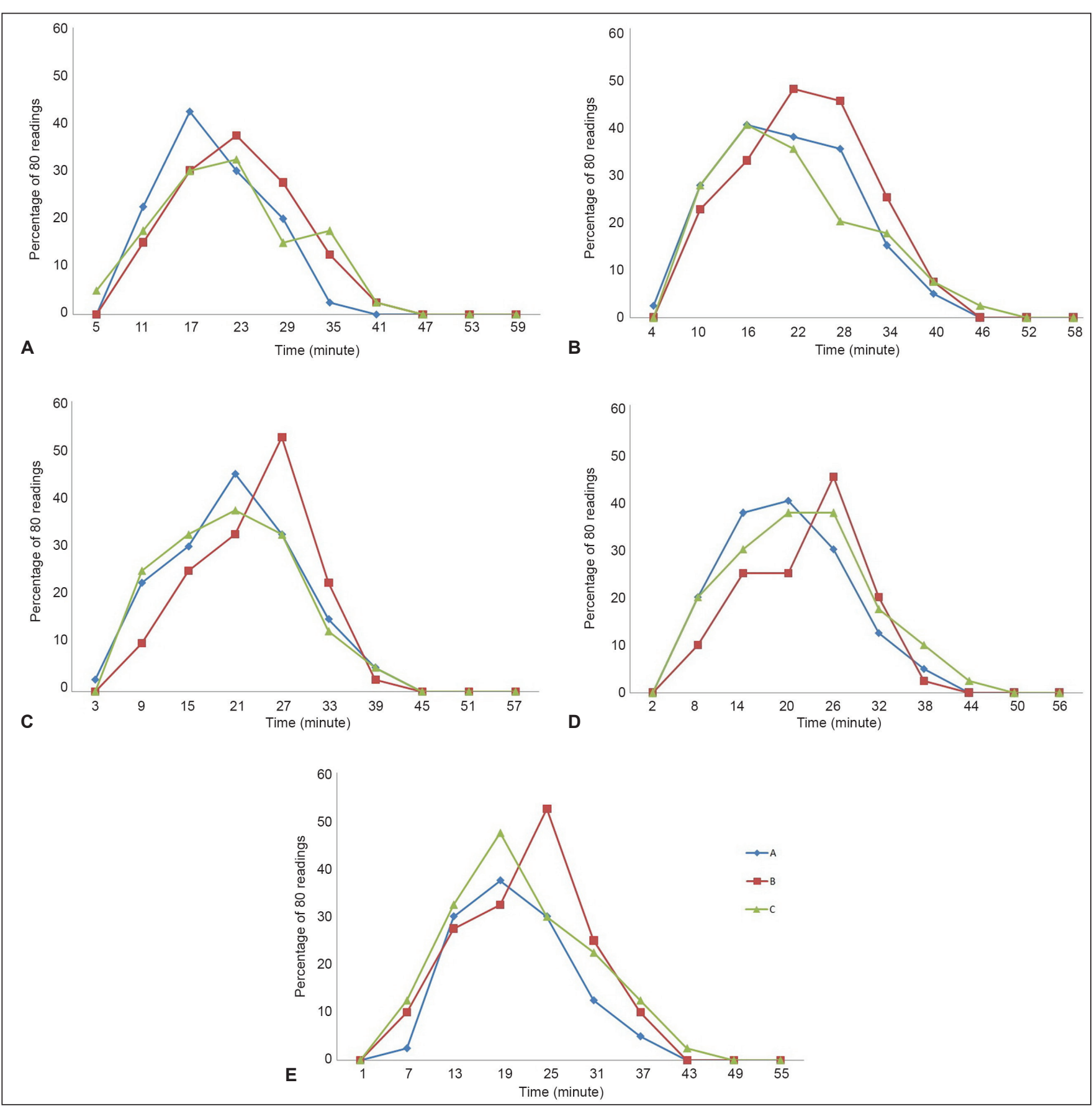

Graphs 1A to E: Pulpal anesthesia of maxillary central incisor (A), lateral incisor (B), canine (C), first premolar (D), and second premolar (E) for each injection site of AMSA nerve block injections. A: Injection at a point bisecting the canine and first premolar, halfway between the midpalatine raphe and the crest of the free gingival margin; B: Injection at a point bisecting the premolars, halfway between the midpalatine raphe and the crest of the free gingival margin; C: Injection at a point bisecting the second premolar and first molar, halfway between the midpalatine raphe and the crest of the free gingival margin; AMSA: anterior middle superior alveolar

\section{DISCUSSION}

The ideal injection for maxillary teeth should result in the rapid onset of pulpal anesthesia for multiple teeth after a single needle penetration. It should not produce collateral anesthesia, and it should only require a minimum dosage of anesthetic solution to be effective. It should be easily administered without any risk to any vital structures. ${ }^{8}$ The AMSA nerve block injection is a promising technique for anesthesia of multiple teeth in the maxilla. It can particularly be useful in patients with systemic diseases, in which the anesthesia of multiple teeth is required and the anesthetic dose should be taken into account. ${ }^{8}$ In the present study, the efficacy of this injection was evaluated in three different sites of the palate.

The results of the present study showed that changing the AMSA nerve block injection site does not 
increase the success rate of this injection. However, as presented in Table 1, the posterior injection site results in a higher success rate of anesthesia for the posterior teeth, demonstrating that injection at the posterior site might provide better anesthesia of the middle superior alveolar nerve branch compared with the conventional site. On the other hand, the anterior injection site results in a higher success rate of anesthesia for the anterior teeth, except for the central incisor, revealing that injection at the anterior site might provide better anesthesia of the anterior superior alveolar nerve branch compared with the conventional site. However, in the present study, as reported in previous studies, ${ }^{16-18}$ the overall success rate of AMSA nerve block injection is low to moderate. This might be due to anatomic variations of infraorbital nerve branches. Anatomical investigations on dissection of cadavers have shown the presence of the middle superior alveolar nerve branch in the range of $30-72 \%$, and when it is absent, its innervation is supplied from a plexus formed by the anterior and posterior superior alveolar branches of the infraorbital nerve. ${ }^{9,10,12}$ The exact role of the absence of the middle superior alveolar nerve branch in the success rate of AMSA nerve block injection is not known. ${ }^{17}$

A general agreement in the studies evaluating the AMSA nerve block injection is about ineffectiveness of this technique for the anesthesia of the central incisors. ${ }^{16-18}$ The results of the present study showed that the AMSA nerve block injection led to mild anesthesia of the central incisors, which is consistent with the results of previous studies, which might be explained by the theory of innervation from the opposite side. ${ }^{19}$

The results of the present study revealed a slow onset of anesthesia for the AMSA nerve block injection, consistent with the results of studies performed by Fukayama et al, ${ }^{16}$ Lee et al, ${ }^{18}$ and Velasco and Soto. ${ }^{17}$ The slow onset of pulpal anesthesia might be attributed to the time necessary for the anesthetic solution to pass through the nutrient canals and reach the AMSA nerve plexus.

Some studies ${ }^{16-18}$ have shown that pulpal anesthesia gradually wears off in 60 minutes, consistent with the results of the present study. However, Friedman and Hochman ${ }^{8}$ claimed that the duration of profound pulpal anesthesia obtained by the AMSA nerve block injection is 45-60 minutes, which is not consistent with the results of the present study (Graph 1A to E).

During the study, three and four subjects did not continue cooperation after the first and second trials, respectively, and left the study due to intolerable pain during injection (Flow Chart 1). One of the main disadvantages of this nerve block injection is that palatal injections are generally painful. ${ }^{20}$ Wahl et $\mathrm{al}^{21}$ showed that palatal injections resulted in significantly more pain than other intraoral injections, probably due to the result of pressure. However, in the present study, to reduce pain during needle insertion and anesthetic solution deposition, the topical anesthesia was applied before each injection and the anesthetic solution was injected slowly.

Evidence $^{22,23}$ shows that lack of response to the maximum output of EPT indicates pulpal anesthesia in vital asymptomatic teeth. In addition, EPT has been used to assess the pulpal anesthesia in previous clinical studies, using local anesthetics. ${ }^{24-28}$ Therefore, a reading of 80 as the maximum output of the EPT was used in the present study as the criteria for pulpal anesthesia.

Some studies have shown no significant differences in the success rate, ${ }^{29}$ onset, $^{29}$ and injection-related pain ${ }^{30}$ of anesthesia between $2 \%$ lidocaine solution with 1:100,000 epinephrine and 3\% mepivacaine solution without epinephrine for the second division and palatal-anterior superior alveolar nerve block injections. Therefore, 3\% mepivacaine solution without epinephrine was used in the present study.

Carryout effect happens when the first trial changes the subject's response to the second trial; therefore, a period of time, called washout period, is needed to eliminate the effect of the first trial. Hence, 1 week was considered for the washout period to eliminate the carryout effect.

A limitation of this study was the mean age of the participants: 26.7 years. Therefore, it might not be possible to generalize the results to children and the elderly.

\section{CONCLUSION}

Under the conditions of the present study, changing the injection site anteroposteriorly did not influence the success rate of the AMSA nerve block injection.

\section{REFERENCES}

1. Loetscher CA, Melton DC, Walton RE. Injection regimen for anesthesia of the maxillary first molar. J Am Dent Assoc 1988 Aug;117(2):337-340.

2. Malamed SF, Trieger N. Intraoral maxillary nerve block: an anatomical and clinical study. Anesth Prog 1983 Mar-Apr; 30(2):44-48.

3. Roda RS, Blanton PL. The anatomy of local anesthesia. Quintessence Int 1994 Jan;25(1):27-38.

4. Mason R, Drum M, Reader A, Nusstein J, Beck M. A prospective, randomized, doubleblind comparison of $2 \%$ lidocaine with 1:100,000 and 1:50,000 epinephrine and 3\% mepivacaine for maxillary infiltrations. J Endod 2009 Sep;35(9): 1173-1177.

5. Gross R, McCartney M, Reader A, Beck M. A prospective, randomized, double-blind comparison of bupivacaine and lidocaine for maxillary infiltrations. J Endod 2007 Sep;33(9): 1021-1024.

6. Haas DA. An update on local anesthetics in dentistry. J Can Dent Assoc 2002 Oct;68(9):546-551. 
7. Gomolka K. The AMSA block: local anesthesia without collateral numbness. CDS Rev 2000 Oct;93(7):34.

8. Friedman MJ, Hochman MN. The AMSA injection: a new concept for local anesthesia of maxillary teeth using a computer-controlled injection system. Quintessence Int 1998 May;29(5):297-303.

9. Fitzgerald M, Scott J. Observations on the anatomy of the superior dental nerves. Br Dent J 1958;104:205-208.

10. Heasman P. Clinical anatomy of the superior alveolar nerves. Br J Oral Maxillofac Surg 1984 Dec;22(6):439-447.

11. Malamed, SF. Handbook of local anesthesia. 4th ed. St. Louis: Mosby; 1997.

12. Mc Daniel WL. Variations in nerve distributions of the maxillary teeth. J Dent Res 1956 Dec;35(6):916-921.

13. Olsen NH, Teuscher GW, Vehe KL. A study of the nerve supply to the upper anterior teeth. J Dent Res 1955 Jun;34(3):413-420.

14. Friedman M, Hochman M. Using AMSA and P-ASA nerve blocks for esthetic restorative dentistry. Gen Dent 2001 Sep-Oct;49(5):506-511.

15. Fischer, G.; Reithmuller, R. Local anesthesia in dentistry. 3rd ed. Philadelphia: Lea \& Febiger;1923.

16. Fukayama H, Yoshikawa F, Kohase H, Umino M, Suzuki N. Efficacy of anterior and middle superior alveolar (AMSA) anesthesia using a new injection system: the Wand. Quintessence Int 2003 Jul-Aug;34(7):537-541.

17. Velasco I, Soto R. Anterior and middle superior alveolar nerve block for anesthesia of maxillary teeth using conventional syringe. Dent Res J (Isfahan) 2012 Sep;9(5):535-540.

18. Lee S, Reader A, Nusstein J, Beck M, Weaver J. Anesthetic efficacy of the anterior middle superior alveolar (AMSA) injection. Anesth Prog 2004;51(3):80-89.

19. Yenisey M.Comparison of the pain levels of computer-controlled and conventional anesthesia techniques in prosthodontic treatment. J Appl Oral Sci 2009 Sep-Oct;17(5):414-420.

20. Hutchins HS Jr, Young FA, Lackland DT, Fishburne CP. The effectiveness of topical anesthesia and vibration in alleviating the pain of oral injections. Anesth Prog 1997 Summer;44(3):87-89.

21. Wahl MJ, Schmitt MM, Overton DA, Gordon MK. Injection pain of bupivacaine with epinephrine vs. prilocaine plain. J Am Dent Assoc 2002 Dec;133(12):1652-1656.
22. Certosimo AJ, Archer RD. A clinical evaluation of the electric pulp tester as an indicator of local anesthesia. Oper Dent 1996 Jan-Feb;21(1):25-30.

23. Dreven LJ, Reader A, Beck M, Meyers WJ, Weaver J. An evaluation of an electric pulp tester as a measure of analgesia in human vital teeth. J Endod 1987 May;13(5):233-238.

24. Chan A, Armati P, Moorthy AP. Pulsed Nd: YAG laser induces pulpal analgesia: a randomized clinical trial. J Dent Res 2012 Jul;91(7 Suppl):79S-84S.

25. Currie CC, Meechan JG, Whitworth JM, Corbett IP. Is mandibular molar buccal infiltration a mental and incisive nerve block? A randomized controlled trial. J Endod 2013 Apr;39(4):439-443.

26. Dressman AS, Nusstein J, Drum M, Reader A. Anesthetic efficacy of a primary articaine infiltration and a repeat articaine infiltration in the incisive/mental nerve region of mandibular premolars: a prospective, randomized, single-blind study. J Endod 2013 Mar;39(3):313-318.

27. Elmore S, Nusstein J, Drum M, Reader A, Beck M, Fowler S. Reversal of pulpal and soft tissue anesthesia by using phentolamine: a prospective randomized, single-blind study. J Endod 2013 Apr;39(4):429-434.

28. Hobeich P, Simon S, Schneiderman E, He J. A prospective, randomized, double-blind comparison of the injection pain and anesthetic onset of 2\% lidocaine with 1:100,000 epinephrine buffered with $5 \%$ and $10 \%$ sodium bicarbonate in maxillary infiltrations. J Endod 2013 May;39(5): 597-599.

29. Forloine A, Drum M, Reader A, Nusstein J, Beck M. A prospective, randomized, double-blind comparison of the anesthetic efficacy of two percent lidocaine with 1:100,000 epinephrine and three percent mepivacaine in the maxillary high tuberosity second division nerve block. J Endod 2010 Nov;36(11):1770-1777.

30. Nusstein J, Burns Y, Reader A, Beck M, Weaver J. Injection pain and postinjection pain of the palatal-anterior superior alveolar injection, administered with the Wand Plus system, comparing $2 \%$ lidocaine with 1:100,000 epinephrine to $3 \%$ mepivacaine. Oral Surg Oral Med Oral Pathol Oral Radiol Endod 2004 Feb;97(2):164-172. 